
Copyright (C) 1996 World Federation for Ultrasound in Medicine \& Biology Printed in the USA. All rights reserved $0301-5629 / 96 \$ 15.00+.00$

PII: S0301-5629(96)00023-3

\title{
TEMPORAL CORRELATION OF BLOOD SCATTERING SIGNALS IN VIVO FROM RADIOFREQUENCY INTRAVASCULAR ULTRASOUND
}

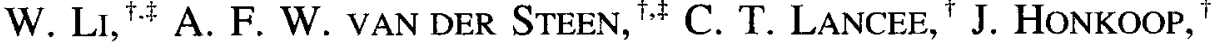 \\ E. J. Gussenhoven ${ }^{\dagger, \ddagger}$ and N. BOM ${ }^{\dagger, \ddagger}$ \\ ${ }^{\dagger}$ Thoraxcentre, Erasmus University Rotterdam and ${ }^{\dagger}$ the Interuniversity Cardiology Institute, \\ Rotterdam, The Netherlands
}

(Received 20 July 1995; in final form 11 December 1995)

\begin{abstract}
One limitation encountered using high frequency intravascular ultrasound (IVUS) is the echogenicity of blood, which increases dramatically at frequencies of 20-40 MHz. Because of the higher velocity of moving blood particles, the echo pattern of flowing blood shows more variations in time than that of the wall. To investigate the time-varying characteristics of the blood scattering, measurements were performed on the radiofrequency (RF) data collected in vivo from five pig experiments. After positioning the echo catheter inside the iliac artery, an M-mode sequence of $30 \mathrm{RF}$ traces was acquired at a high pulse repetition rate $(5 \mathrm{kHz})$. The $\mathrm{RF}$ correlation time was measured on the regions of blood and the arterial wall. Two processing techniques, temporal averaging and correlation, were tested for suppression of the blood echo intensity. The correlation time Tc measured in the blood region was approximately $1 \mathrm{~ms}$, which was shorter than that measured in the wall region ( $\mathrm{Tc} \gg 6 \mathrm{~ms}$ ). The correlation values calculated in a small window showed a large variation in the blood region while the wall region produced a constant high output. After processing eight consecutive RF traces $(\Delta T=200 \mu \mathrm{s}$ ), the temporal averaging method results in a $50 \%$ intensity reduction in the blood region. Using the correlation output as a weighting function, the blood echo intensity can be further reduced to only $10 \%$ of its original value. Application of the RF correlation processing to a cross-sectional image data demonstrates the feasibility of this technique to remove most of the blood echoes and enhance the image contrast of the luminal interface.
\end{abstract}

Key Words: Temporal correlation, Blood scattering, in vivo, Intravascular ultrasound.

\section{INTRODUCTION}

Intravascular ultrasound (IVUS) is a real-time tomographic imaging modality that is being widely used in combination with various types of vascular intervention techniques (Bom et al. 1989; Nissen et al. 1991; The et al. 1992b; Yock et al. 1993). The use of high frequency ultrasound $(20-40 \mathrm{MHz})$ is a key element to achieve high resolution imaging of the arterial anatomy. One implication of using high ultrasonic frequencies is an increase of the blood echogenicity. The intensity of blood scattering may reach a level similar to that of the arterial tissue, leaving little contrast at the luminal interface. With real-time IVUS imaging, it can be seen that the scattering pattern of flowing blood varies over time, whereas echoes of the arterial tissue present themselves as a fixed pattern. This phenomenon allows identification of the blood echoes in real-

Address correspondence to: W. Li, Thoraxcentre (Ee 2302), Erasmus University Rotterdam, P.O. Box 1738,3000 DR Rotterdam. The Netherlands. E-Mail:li@tch.egg.eur.nl time IVUS. Based on the changing characteristics of the blood scattering, we have demonstrated a temporal video averaging method to improve the image quality for off-line quantitative analysis ( $\mathrm{Li}$ et al. 1992, 1994). A similar approach was also reported by Pasterkamp ct al. (1993), whercby an image subtraction technique was applied for enhancement the of the luminal contrast. These video processing techniques, although easy to implement, have two drawbacks. First, since the processing needs at least two vidco frames with a time interval of $20 \mathrm{~ms}$, rapid wall motion may introduce blurring on the real-time image. Second, the image enhancement obtained by video processing is limited by the lack of phase information in the demodulated signals. As the phase shift in the radiofrequency (RF) signals is a sensitive index for motion detection (Bohs et al. 1991; De Jong et al. 1991; Hein et al. 1993; Fcrrara et al. 1994), development of improved image processing methods is therefore directed toward processing of the original RF echo data. To evaluate the feasibility of RF signal processing techniques, the tem- 
poral characteristics of the blood scattering during IVUS imaging needs to be investigated. Gronningsaeter et al. (1995) have studied the effects of Doppler shift and frequency broadening respectively caused by the axial and lateral movements of blood particles in simulated data. An RF correlation detector for blood echo rejection was evaluated in vitro using water mixed with sephadex particles in a tissue-like phantom (Gronningsaeter et al, 1994). The cyclic changes of blood echogenicity in IVUS video signals were reported by De Kroon et al. (1991). Several acoustic properties of blood and wall tissue were measured in vitro by Lockwood et al. ( 1991 ) at frequencies of 25$65 \mathrm{MHz}$. The backscatter level of flowing blood as a function of shear rate has been investigated at different frequency ranges by several groups (Yuan et al. 1988; De Kroon et al. 1993; Shung et al. 1993; Foster et al. 1994). Little information about the temporal properties of the RF blood scattering signals is available from an in vivo IVUS set-up, in which the ultrasound beam is always perpendicular to the direction of blood flow.

The purpose of this study was to investigate the temporal characteristics of the blood scattering in vivo. The measurements were made in animal experiments under normal flow conditions. Two processing techniques, temporal averaging and correlation, were tested on the collected RF data.

\section{MATERIALS AND METHODS}

\section{Experimental set-up}

This study was performed in vivo in the iliac artery of Yorkshire pigs according to the guiding principles for the care and use of animals outlined by the American Physiological Society and approved by the Committee on Animal Experiments of the Erasmus University. Ultrasonic data were acquired using a $30 \mathrm{MHz}$ IVUS imaging system (Du-MED, Rotterdam, The Netherlands) with a single-element transducer mounted on the tip of a $5 \mathrm{~F}$ catheter. Cross-sectional images were scanned by means of a flexible drive shaft rotation. The bandwidth of the system was $20-40 \mathrm{MHz}$, yielding an axial resolution of approximately $75 \mu \mathrm{m}$. During the experiments, the echo catheter was advanced through a $7 \mathrm{~F}$ sheath into the iliac artery. At the site of interest, a few seconds of crosssectional IVUS images were first recorded on the videotape as a reference. Keeping the catheter at the same position, the imaging system was then switched to $\mathrm{M}$ mode. The orientation of the transducer was manipulated to select an angular position at which the scan line contained the echoes from both blood and the arterial wall.

\section{Data acquisition}

The RF data acquisition set-up is illustrated in Fig. 1. At each selected angular position, a burst of 30 ultrasound beams was transmitted. The pulsc repetition rate was $5 \mathrm{kHz}$. Data acquisition was manually initialized at an arbitrary moment of the cardiac cycle. The received RF signal was amplified $40 \mathrm{~dB}$ by the amplifier of the imaging system and digitized by a dual channel digital oscilloscope (LeCroy 9400, Chestnut Ridge, NY, USA). The sampling was performed at a frequency of $200 \mathrm{MHz}$ with 8 bits resolution. The sample duration of RF traces was $10 \mu \mathrm{s}$, yielding a scan depth of approximately $8 \mathrm{~mm}$. To avoid jitter between RF traces, the pulse generator was synchronized with the internal sampling clock of the oscilloscope. The digital data were transferred to the host computer via an IEEE connection. The data acquisition procedure was controlled by a PC-486 with in-house developed software.

Measurements were performed in five pig experiments. In each experiment, three different angular positions were selected. RF signals were acquired three times at the same angle to obtain data from different phases of a cardiac cycle. A total of 45 measurements containing $1350 \mathrm{RF}$ traces became available for further analysis.

\section{Temporal correlation}

A temporal correlation method was used to evaluate the changes in the RF trace sequence acquired from the same angle with a time interval of $200 \mu \mathrm{s}$. The correlation coefficient $R(n)$ hetween the first RF trace So and the subsequent $n$th trace $\mathrm{Sn}$ was determined by the following formula:

$$
\begin{aligned}
& R(n)=\frac{\sum_{i=1}^{K}[\operatorname{So}(i)-\overline{\operatorname{So}}][\operatorname{Sn}(i)-\overline{\mathrm{Sn}}]}{\sqrt{\sum_{i=1}^{K}[\operatorname{So}(i)-\overline{\operatorname{So}}]^{2} \sum_{i=1}^{K}[\operatorname{Sn}(i)-\overline{\operatorname{Sn}}]^{2}}} \\
& \mathrm{n}=1,2 \ldots 29
\end{aligned}
$$

Where $n$ is the index of the RF trace, $K$ is the number of data points in a correlation window and $i$ is the index of the data points. $\overline{\text { So }}$ and $\overline{\mathrm{Sn}}$ are the means of the RF data in the window. This procedure yielded a series of $\mathbf{R}$ values ranging from -1 to 1 , representing the similarity between the RF trace arriving at To and that arriving at $\operatorname{Tn}=\mathrm{n} * 200 \mu \mathrm{s}$.

\section{Data processing I: RF correlation measurements}

Correlation curve in a region. The $30 \mathrm{RF}$ traces acquired at one angular position (indicated by a white line in Fig 2) were peak-detected and displayed as an M-mode image. The motion of the blood particles was identified on the M-mode display (Fig 2). The luminal region showed a random pattern plus some diagonal 


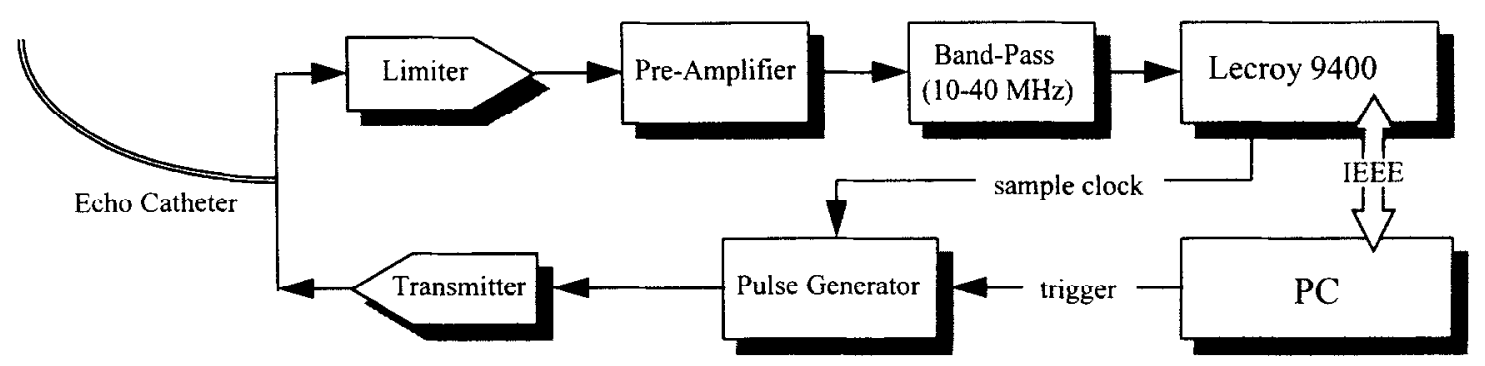

Fig. 1. Block diagram of the RF data acquisition set-up.

streaks produced by motion components parallel to the ultrasound beam. The echoes from the arterial tissue presented as a stationary pattern throughout the acquisition period (Fig 2). Using this as a visual criterion, the $\mathrm{RF}$ traces were manually divided into two regions: blood and wall. The B-mode images recorded at the same catheter position were reviewed during the data processing. Anatomic structures such as the adjacent vein were identified to avoid improper selection of the region of interest. The time-varying characteristics of the RF signals were evaluated using the correlation method from these selected blood and vessel wall regions. For each region, the correlation coefficient was calculated using equation 1 where the parameter $\mathrm{K}$ was set to the size of the region. The number of available data points ranged from $200-1000$, depending on the lumen dimension for the blood region and the wall thickness for the tissue region. The correlation curves of the blood and wall regions were obtained from the mean values of the 45 regional measurements at each delay time. The point at which the correlation coefficient dropped to a value $\leq 0.1$ in the curve was defined as the correlation time $\mathrm{Tc}$.

Correlation distribution in a window. To investigate the possibilities of using correlation techniques for image enhancement, the behavior of the correlation procedure was studied in a small window. When equation 1 is applied to suppress the blood signals, the correlation output needed to have a time resolution comparable to that of the B-mode image. The window size $\mathrm{K}$ for the correlation calculation was set to seven points to provide a sufficient resolution. The time period of the window was $35 \mathrm{~ns}$ and the length was $28 \mu \mathrm{m}$. This window length was approximately one acoustic wavelength of the imaging system, which ensured no smearing of the image was introduced by the correlation procedure. After the region separation, the data were subdivided into a sequence of non-overlapping windows. A similar correlation process as described above was performed in the window data. The correlation output range $(-1-1)$ was divided into 20 bin ranges with intervals of 0.1 . For both the blood and tissue re- gions, distribution histograms at each delay time were calculated from the whole data set.

\section{Data processing II: RF image processing}

Methods for image enhancement. Two processing methods, temporal averaging and correlation, were tested on experimental data. The first method was to average a number of RF traces. Because of the randomness in the blood scattering signals, the averaging procedure was expected to reduce the signal amplitude. The second method was to use the correlation output as a weighting function to modulate the RF signals. Figure 3 illustrates the correlation method by an example with a sequence of 8 RF traces. First, a number of RF traces was selected from the original RF traces (Fig. 3a) and a temporal averaged RF trace was obtained (Fig. 3b). Secondly, a moving window with a length of seven points was defined along the depth axis of an RF trace. The interval between two adjacent windows was three points yielding $50 \%$ overlap. Third, a sequence of correlation values $\mathrm{R}$ was obtained at each window position using equation 1 . From this sequence, the minimum value was selected as the weighting factor for that particular window position. A weighting curve was generated from all available window positions (Fig. 3c); a high value in the weighting curve indicates no motion was detected, whereas a low value indicates changes had occurred within the acquisition time. Finally, the output signal was obtained by multiplying the averaged signal with its corresponding weighting factor (Fig. 3d).

Quantitative evaluation of the RF methods. The effect of these methods was quantitatively evaluated by comparing the echo intensity in the blood region before and after processing. The echo intensity of the blood region was defined as the mean value of the envelope signal obtained by the Hilbert transform. The echo intensity derived from the first RF trace was used as a reference to estimate the original intensity before processing. The relative intensity changes after processing a number of subsequent RF traces were measured with the ratio between the processed intensity and the intensity of the first RF trace. 

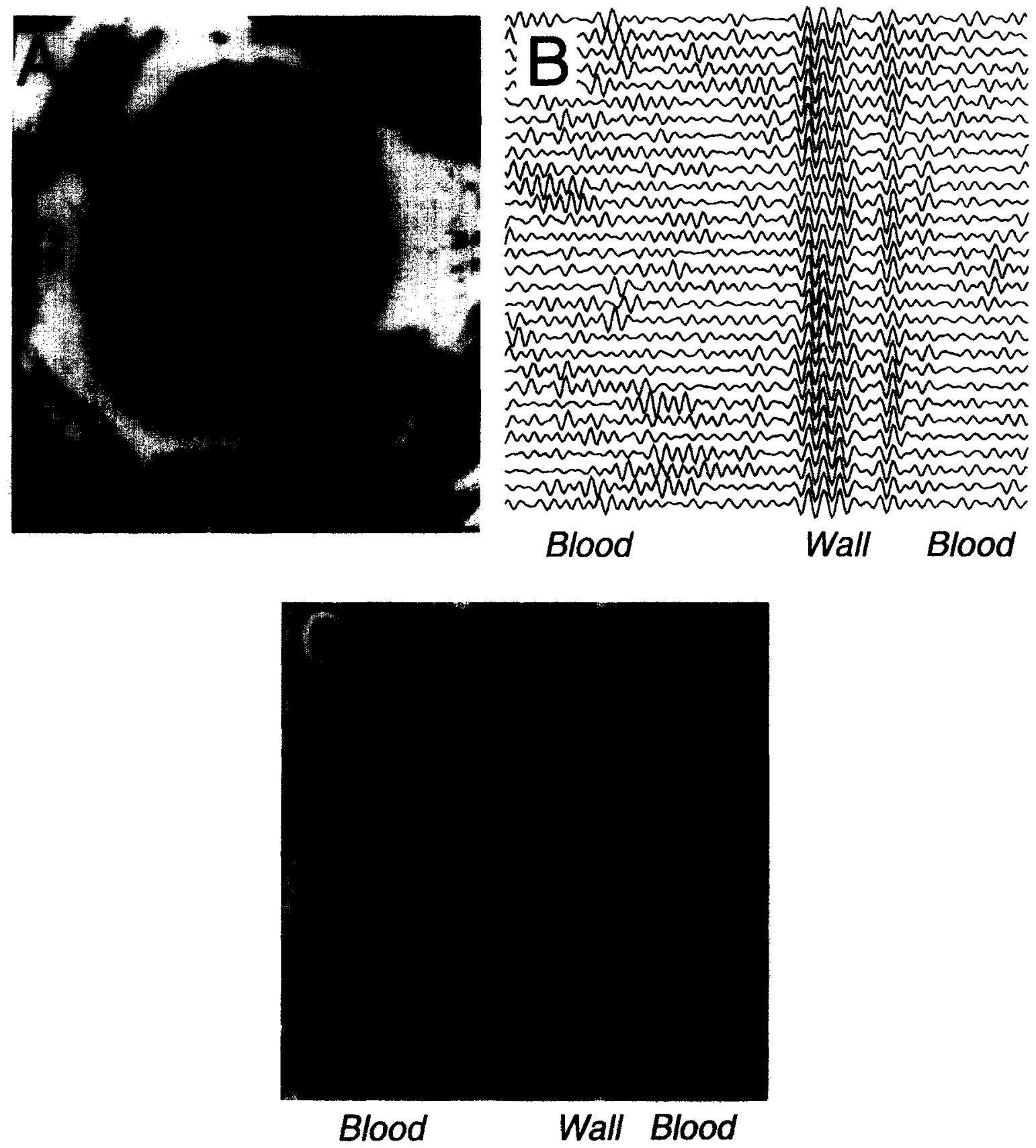

Fig. 2. Illustration of RF data acquisition. a) An iliac cross-section obtained by the IVUS imaging system. b) RF sequence acquired at one angle. The angular position of the transducer is indicated by the white line in (a). c) Corresponding $\mathrm{M}$-mode display. The gray-scale on the $\mathrm{M}$-mode image was log compressed. Note the changing structure of the blood region in contrast to a fixed pattern in the wall. The random and diagonal patterns were caused by the transverse and axial movements of blood particles, respectively.

Qualitative evaluation of the $R F$ methods. To verify the visual improvement by the RF techniques, another experiment was performed to obtain a complete crosssectional scan. Because of the limitation in data storage capability, the current set-up was not able to acquire cross-sectional RF data in real-time. A stepper-motor device was used to rotate the transducer at a slow speed in synchronism with the blood pressure. The pressure gating ensured that RF data were acquired from the same phase of the cardiac cycle, and thus minimized the motion artifact for off-line image reconstruction. The cross-sectional scan was obtained in 200 angular steps. At each angle, ultrasonic data were acquired in a similar way as abovedescribed. The averaging and correlation processing were performed on the first eight RF traces at each step. The resulting data were scan-converted and displayed as a 


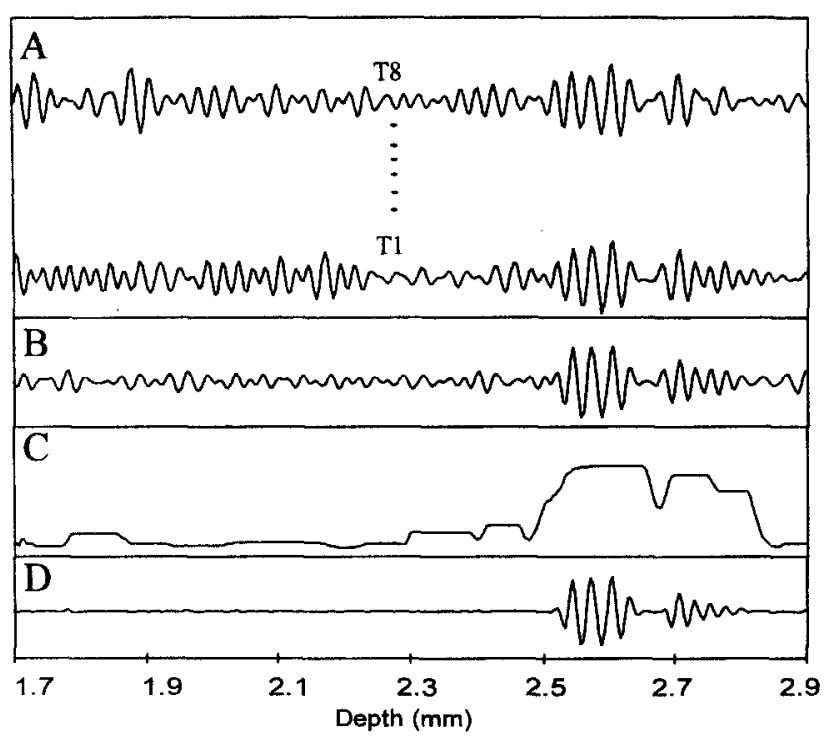

Fig. 3. Example of RF processing from data obtained at one angle. a) An original $R F$ sequence with eight traces. b) Averaged output of the RF sequence. $c$ ) Weighting function derived by the correlation method. d) Processed trace obtained by multiplying (b) and (c).

gray-scale image. Logarithmic compression was applied to the image data allowing the resulting display to be comparable to that from a conventional IVUS imaging system.

\section{RESULTS}

\section{Correlation curve}

The mean correlation values of the regional measurements are plotted as a function of the delay in Fig. 4. The correlation curve in the wall region declines slowly as the delay increases and maintains a high value $(>0.8)$ until the end of the data acquisition period. The correlation output of the blood region, in contrast, drops rapidly as the delay increases. The mean correlation value is $<0.4$ between the first and second RF traces. The correlation time of the blood region is approximately $1 \mathrm{~ms}$. Although it is not possible to measure the correlation time of the wall region due to the short data acquisition period, it may be predicted that $\mathrm{Tc}$ will be $\gg 6 \mathrm{~ms}$ from the trend of the curve.

\section{Correlation distribution}

The distribution of the correlation values calculated in the small window is shown in Fig. 5. In the wall region, the outputs are mostly concentrated around the high correlation values and spread slowly as the delay increases. The values from the blood region show an almost random distribution in the whole correlation range. The high variability in the correla- tion output suggests that correlating only two RF traces may still produce a high value at some locations of the blood region. Therefore, more traces are needed for a reliable rejection of the blood echoes.

\section{Intensity changes by $R F$ processing}

Figure 6 illustrates the intensity decrease in the blood region resulting from the temporal averaging and correlation processing. The results show averaging a number of RF traces is able to reduce the intensity of the blood echoes. Averaging eight sequential RF traces results in approximately $50 \%$ decrease of the echo intensity. Only a small improvement can be gained by averaging more than eight traces. By applying the correlation procedure in eight $R F$ traces, the signal intensity of blood was reduced to $<10 \%$ of its original intensity.

\section{Image enhancement by $R F$ processing}

The results of the image enhancement by processing eight RF traces from the cross-sectional image data are illustrated in Fig. 7. The unprocessed image was constructed from the first trace of each RF sequence (Fig. 7a). As can be seen, strong blood scattering echoes obscure the visibility of the luminal boundary. The luminal intensity was reduced slightly by averaging eight sequential RF traces (Fig. $7 \mathrm{~b}$ ). The actual visual enhancement was partly reduced by the use of logarithmic compression during video display. Figure $7 \mathrm{c}$ displays the weighting function derived by the correlation method. The image shows a binary structure with white (1) for tissue and value black (0) for blood. The image processed by multiplying Figs.

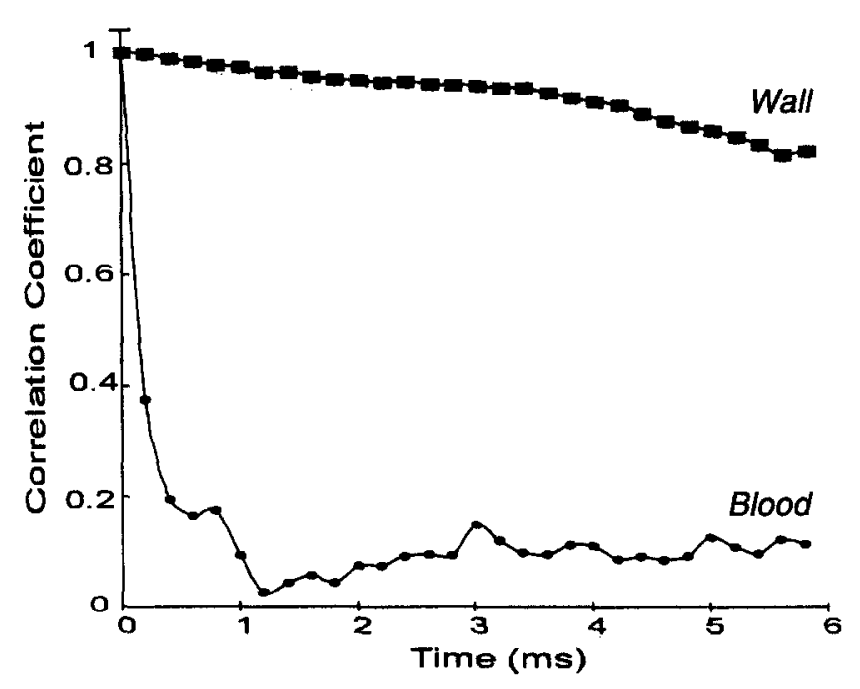

Fig. 4. Mean correlation values determined in the two regions. Note a steep drop in the correlation curve of the blood region as compared to a slow decrease in the correlation curve of the wall region. 


\section{In Blood Region}

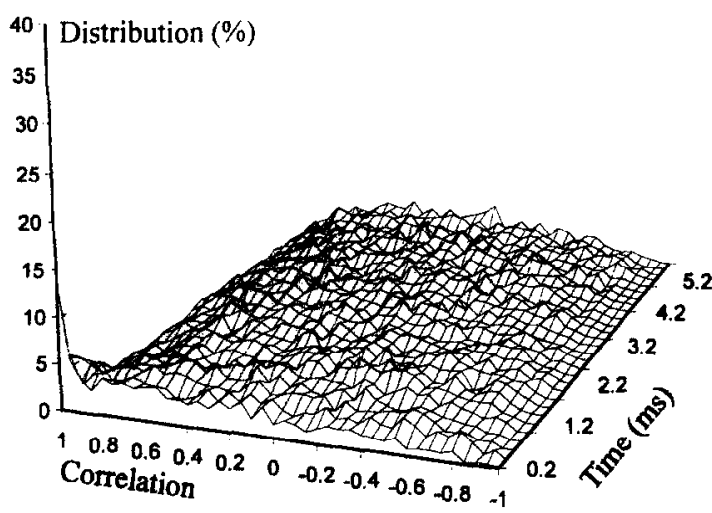

In Wall Region

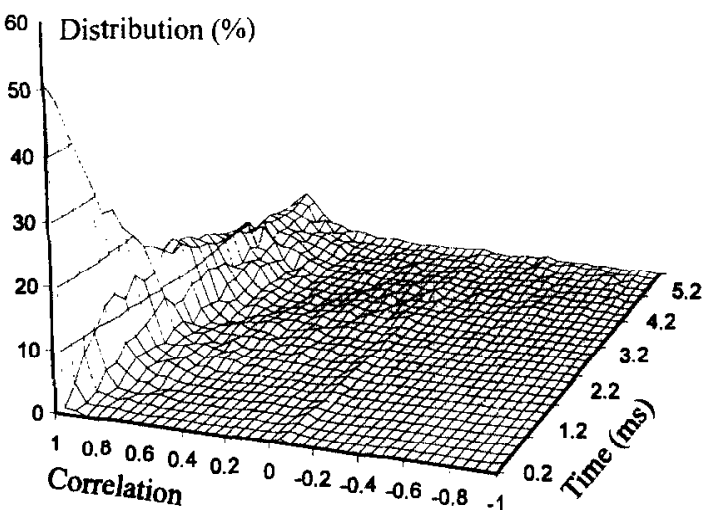

Fig. 5. Distribution of the correlation values calculated in a non-overlapping window of seven points. Note the randomness of the correlation outputs in the blood region for all delay time.

$7 \mathrm{~b}$ and $\mathrm{c}$ shows a significant improvement in the image contrast between the lumen and wall (Fig. 7d). The blood scattering echoes were mostly removed while the details in the arterial wall were preserved.

\section{DISCUSSION}

Results of this study show the differences in the correlation properties between blood and vessel wall. The movement of blood particles can be separated into two velocity components, the transverse and axial flows, in relation to the ultrasound beam direction. The transverse flow causes blood particles to move in and out of the ultrasound beam while the axial flow moves blood particles along the ultrasound beam. The transverse and axial flows will alter the correlation output of the blood signals in different manners. In the case

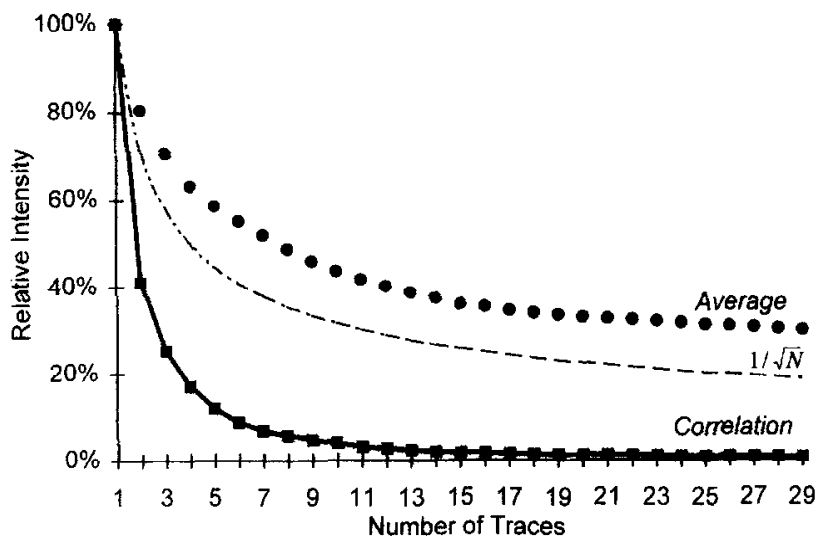

Fig. 6. Intensity decrease resulting from the averaging and correlation methods. The theoretical reduction curve $(1 / \sqrt{N}, N$ is number of traces) of averaging completely uncorrelated RF sequences plotted as dotted line for comparison. of the transverse flow, the signals generated by the ncwly moving-in particles will be uncorrelated with those generated by the particles staying inside the ultrasound beam because of the random distribution of blood particles. Thus, the correlation time can be estimated from the beam width divided by the flow velocity (Bonnefous 1989). The beam width of the IVUS systcm used is $1 \mathrm{~mm}$. Assuming the mean blood velocities for the normal peripheral flow are in the range of $40-80 \mathrm{~cm} / \mathrm{s}$, the time required by blood particles to

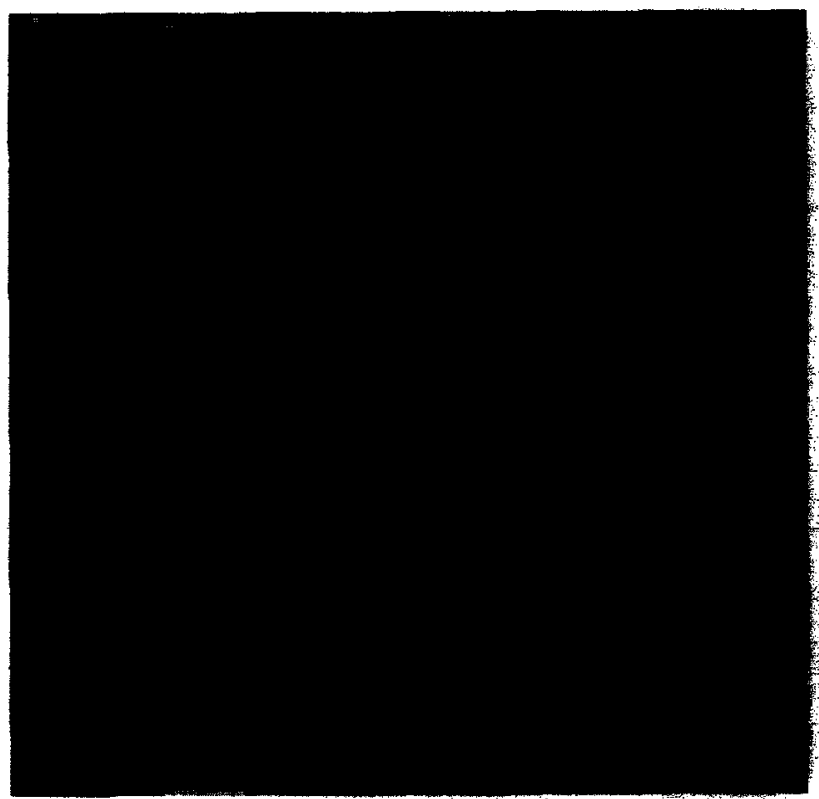

Fig. 7. Example of RF processing from the cross-sectional image data. a) Image constructed from the first RF trace of each angle. b) Result after averaging of eight RF traces per angle. c) Image constructed from the correlation weighting function obtained from eight RF traces per angle. d) Image processed by multiplying (b) and (c). 
travel through the ultrasound beam will be in the order of milliseconds. The measured $\mathrm{Tc} \approx 1 \mathrm{~ms}$ falls within the range of this estimation. As the transducer is placed perpendicular to the vessel wall in the IVUS setting, the transverse velocity is cxpected to be a main factor for the decrease of the correlation time. On the other hand, the presence of the echo catheter may cause the development of the axial velocity in part of blood flow. The axial movement of blood particles can be observed from the diagonal moving structure on the M-mode image in Fig. 2. The axial flow component introduces a phase shift between the two consecutive RF signals. The relation between the correlation output and the phase shift is determined by the autocorrelation curve of the system response. In a typical situation, an axial displacement of $12 \mu \mathrm{m}$ may yield $180^{\circ}$ phase shift and a correlation value of -1 . For two RF traces separated by $200 \mu \mathrm{s}$, the equivalent axial velocity will be $6 \mathrm{~cm} /$ $\mathrm{s}$. The negative correlation was not detected in the correlation curve because the influence of the transverse flow was the dominant factor when measuring the mean value in the whole blood region. In the small window, the phase shift effect can be appreciated from Fig. 5, where the correlation values of the blood region scatter randomly over the range from -1 to 1 . The slow decrease of the correlation output measured in the wall region was probably caused hy the wall pulsation producing motion in line with the ultrasound beam. Measurements on wall distensibility with IVUS have exhibited 5\% (The et al. 1992a) and 18\% (Nissen et al. 1991 ) luminal area changes during the cardiac cycle for the peripheral and coronary arteries, respectively. When translating this information to wall velocities, the maximum axial velocity of the vessel wall will be lower than $1 \mathrm{~mm} / \mathrm{s}$.

Temporal averaging is a simple method to reduce the amplitude of a random signal. Assuming the blood signals to be wide-band random noise, the averaged intensity output is expected to be reduced by the square root of the number of the RF traces used. A similar trend to that predicted by the square root reduction can be observed by comparing the two curves in Fig. 6. The averaging effect obtained in the present data set, however, is lower than the square root reduction because the RF sequences used for average are still partly correlated. The correlation weighting function, on the other hand, is able to produce a near zero value whenever the signals are uncorrelated. Combination of the averaging and correlation methods has shown to be more efficient; the blood echoes can almost be rejected by processing eight RF traces. Real-time implementation of the correlation processing, however, will require sophisticated digital circuits with high processing speed and large storage capacity. Another consideration in real-time application is the timing design for the cross-sectional scan. The correlation procedurc requires a prolonged scan time at each beam position in order to collect sufficient changes in the blood scattcring signals. This will be at the expense of either a reduccd frame-rate or a lower angular resolution. For the example given in Fig. 7 , the time required for each beam position is $1.6 \mathrm{~ms}(8 \times 200 \mu \mathrm{s})$. In order to achieve 200 angular positions in one cross-sectional image, the frame-rate will be reduced to $2.8 \mathrm{frame} / \mathrm{s}$, which is too low to display rapid motion of the arterial wall or flaps. One method to increase the frame-rate is to reduce the number of scan lines by performing image interpolation between scan lines. Another solution is to extend the correlation procedure by applying a multidimensional correlator on a sequence of RF data blocks. A multidimensional processing may take advantages of the blood scattering properties in both the time and spatial domains to reduce the waiting period at one beam position.

This study was performed in the peripheral artery with normal blood flow. The results may be applicable to intracoronary imaging as the coronary flow has approximately the same velocity ranges as that of the peripheral arteries. The influence of a lesion with a reduced flow velocity was not included in this study. Since the M-mode RF data were collected randomly from different cardiac phases, the measurement results presented an averaged effect of a pulsatile flow. In practice, the effectiveness of the correlation processing depends mainly on the differences between the dynamics of flowing blood and wall motion.

\section{CONCLUSION}

High blood echogenicity in IVUS imaging has been a bottle-neck for accurate determination of the luminal boundary, particularly for computerized quantification. Information about the time-varying characteristics of the blood scattering is essential for improving the IVIIS image quality. This study indicates that the RF correlation time is significantly shorter in the blood region ( $\mathrm{Tc} \approx 1 \mathrm{~ms}$ ) than that measured in the wall region ( $\mathrm{Tc} \gg 6 \mathrm{~ms}$ ). The fast decorrelation in the blood scattering signals allows an RF processing to be performed without smearing dynamic wall motion in real-time IVUS imaging. A simple temporal RF averaging is able to reduce $50 \%$ of the original blood echo intensity. The proposed correlation technique improves the blood suppression up to a $90 \%$ intensity reduction. and thus provides a satisfactory contrast enhancement for the lumen interface. Furthermore, the correlation weighting function can be incorporated into an automatic contour detection algorithm for real-time extraction of the luminal boundary.

Acknowledgement - These investigations are supported by grant RGN44.3462 of the Netherlands Technology Foundation (STW). 


\section{REFERENCES}

Bohs LN. Trahey GE. A novel method for angle independent ultrasonic imaging of blood flow and tissue motion. IEEE Trans Biomed Eng 1991;3:280-286.

Bom N, ten Hoff H, Lancée CT, Gussenhoven EJ, Bosch JG. Early and recent intraluminal ultrasound devices. Int J Cardiac Imag 1989:4:79-88.

Bonnefous $O$. Statistical analysis and time correlation processes applied to velocity measurement. IEEE Ultrason Symp Proc 1989:887-892.

De Jong BGM, Arts T, Hoeks APG, Reneman RS. Experimental evaluation of the correlation interpolation technique to measure regional tissue velocity. Ultrason Imag 1991;13:145-161.

De Kroon MGM. Acoustic backscatter in arteries. Ph.D. Thesis. Erasmus University, Rotterdam, The Netherlands: 1993.

De Kroon MGM, Slager CJ, Gussenhoven WJ, et al. Cyclic changes of blood echogenicity in high-frequency ultrasound. Ultrasound Med Biol 1991; 17:723-728.

Ferrara KW. Zagar B, Algazi R, Silverman R, Sokil-Melgar J. An experimental evaluation of the received signal from blood at 50 MHz. IEEE Ultrason Symp Proc 1994:1603-1607.

Foster FS, Ohara H, Bloomfield T, Ryan IJK, Lockwood GR. IJltrasound backscatter from blood in the 30 to $70 \mathrm{MHz}$ frequency range. IEEE Ultrason Symp Proc 1994:1599-1602.

Gronningsaeter A, Angelsen BAJ, Gresli A, Torp H. Blood noise reduction in intravascular ultrasound imaging. IEEE Trans Ultrason Ferroelec Freq Contr 1995;42:200-208.

Gronningsaeter A, Angelsen BAJ, Heimdal A, Torp H. Vessel wall detection and blood noise reduction in intravascular ultrasound imaging. IEEE Ultrason Symp Proc 1994:1609-1612.

Hein A, $O$ Brien D. Current time-domain methods for assessing tissue motion by analysis from reffected ultrasound echoes - A review. IEEE Trans Ultrason Ferroelec Freq Contr 1993;40:84102.
Li W, Bosch JC, Gussenhoven WJ, et al. Semiautomatic frame-loframe tracking of the luminal border from intravascular ultrasound. Proc Computers Cardiol 1992:353-356.

Li W, Gussenhoven EJ, Zhong Y, et al. Temporal averaging for quantification of lumen dimensions in intravaseular ultrasound images. Ultrasound Med Biol 1994;20:117-122.

Lockwood GR, Ryan LK, Hunt JW, Foster FS. Measurement of the ultrasonic properties of vascular tissues and blood from 35-65 MHz. Ultrasound Med Biol 1991; 17:653-666.

Nissen SE, Curley JC. Application of intravascular ultrasound for detection and quantitation of coronary atherosclerosis. Int $\mathbf{J}$ Card Imag 1991;6:165-177.

Pasterkamp G, van der Heiden MS, Post MJ, et al. Discrimination of intravascular lumen and dissections in a single $30 \mathrm{MHz}$ ultrasound image: Use of 'confounding' blood backscatter to advantage. Radiology 1993; 187:871-872.

Shung KK, Kuo IY, Cloutier G. Ultrasonic scattering properties of blood. In: Roelandt J, Gussenhoven EJ, Bom N, eds. Intravascular Ultrasound Inc. Dordrecht, Kluwer Academic Publishers: 1993:79-90.

The SHK, Gussenhoven EJ, Li W, et al. Intravascular ultrasonic assessment of lumen geometry and distensibility of the angiographically normal artery: A correlation with quantitative angingraphy. Echocardiography 1992a;9:133-139.

The SHK, Gussenhoven EJ, Zhong Y, Li W, van Egmond F. The effect of balloon angioplasty on the femoral artery cvaluated with intravascular ultrasound imaging. Circulation 1992b; 86:483-493.

Yock PG, Fitzgerald PJ, Sudhir K. Intracoronary ultrasound scanning - Clinical experience and new insights. In: Roelandt J, Gussenhoven EJ, Bom N, eds. Intravascular Ultrasound, Dordrecht, Kluwer Academic Publishers: 1993:17-32.

Yuan YW, Shung KK. Ultrasonic backscatter from flowing blood. I. Dependency on shear rate and hematocrit. J Acoust Soc Am 1988:84:52-58. 